\title{
EchoGéo
}

42 | 2017

Varia

\section{Où accueillir les mineurs non accompagnés en France ? Enjeux de la construction d'un territoire de protection}

Sarah Przybyl

\section{CpenEdition}

Journals

Édition électronique

URL : https://journals.openedition.org/echogeo/15162

DOI : 10.4000/echogeo.15162

ISSN : 1963-1197

Éditeur

Pôle de recherche pour l'organisation et la diffusion de l'information géographique (CNRS UMR 8586)

Référence électronique

Sarah Przybyl, « Où accueillir les mineurs non accompagnés en France ? Enjeux de la construction d'un territoire de protection », EchoGéo [En ligne], 42 | 2017, mis en ligne le 31 décembre 2017,

consulté le 31 juillet 2021. URL : http://journals.openedition.org/echogeo/15162 ; DOI : https://doi.org/ 10.4000/echogeo.15162

Ce document a été généré automatiquement le 31 juillet 2021.

EchoGéo est mis à disposition selon les termes de la licence Creative Commons Attribution - Pas d'Utilisation Commerciale - Pas de Modification 4.0 International (CC BY-NC-ND) 


\title{
Où accueillir les mineurs non accompagnés en France ? Enjeux de la construction d'un territoire de protection
}

\author{
Sarah Przybyl
}

\section{Introduction}

1 Depuis près de trente ans, en France métropolitaine, l'arrivée croissante de mineurs non accompagnés ${ }^{1}$ met au défi les institutions et bouscule les logiques de gestion territoriale de la protection de l'enfance. Dès la fin des années 1990, ces jeunes ont été érigés en une problématique à la croisée entre gestion de l'immigration, protection de l'enfance en danger et considérations politiques. Venus des pays d'Afrique de l'ouest, du Maghreb, d'Asie ou de l'Europe de l'est, ces individus de moins de 18 ans ont emprunté les routes de la migration et se trouvent sur le territoire français sans leur représentant légal. Âgés de 15 à 17 ans, ils étaient 8054 à avoir été reconnus non accompagnés pour l'année 2016 (Ministère de la justice, 2017). À l'image d'autres flux migratoires, le voyage des mineurs ne se termine pas n'importe où sur le territoire. Dans ce contexte, des agglomérations et des départements sont plus concernés que d'autres par leur arrivée. Cette inégale répartition départementale des mineurs non accompagnés révèle le jeu d'acteurs institutionnels pris au cœur d'enjeux territoriaux de protection de l'enfance.

2 Alors que les publications académiques et associatives consacrées à leur situation sociale et juridique se multiplient, les géographes ont encore peu analysé cette réalité du point de vue des dynamiques spatiales qu'elle présente. Comme le soulignent Eugénie Terrier et Juliette Halifax (2017), les approches territoriales de la protection de l'enfance sont à cet égard encore peu nombreuses. Cette contribution de géographie retrace les étapes de la construction d'un territoire dérogatoire de la protection de 
l'enfance pour analyser les évolutions spatiales de leur protection. Sorte de petite décentralisation dans la grande, l'accueil des mineurs non accompagnés révèle le déploiement des dispositifs originellement dérogatoires à l'échelle nationale.

3 Les éléments présentés dans cet article reposent sur une recherche doctorale (Przybyl, 2016) menée entre 2012 et 2016, articulant des phases d'immersion dans plusieurs espaces de la prise en charge des mineurs non accompagnés en France métropolitaine. Dans les lieux d'accueil d'urgence des grandes villes (Paris, Marseille et Montpellier) ou encore dans le huis clos des foyers d'accueil au sein de communes rurales (Treignac en Corrèze, Saverdun en Ariège), la mise en place d'un dispositif d'enquête qualitatif a permis d'appréhender les réalités des contours de la protection. Pour recueillir le point de vue des mineurs comme celui des professionnels (chefs de service, éducateurs spécialisés, stagiaires, professeurs de français, etc.), des entretiens semi-directifs (13 avec des mineurs, 14 avec des professionnels), des échanges informels, des observations journalières ou encore la réalisation d'ateliers vidéo ont jalonné les temps de l'investigation². Âgés de 13 à 17 ans, c'est pour mieux refléter la diversité des origines des mineurs enquêtés qu'aucune sélection de nationalité n'a été opérée. En plus d'un engagement au plus près des protagonistes, le suivi des actualités sur cette thématique a ouvert la voie à une appréciation des réalités d'autres localités mais aussi à l'appréhension des évolutions du traitement politique de ce sujet.

\section{Quand les mineurs non accompagnés déséquilibrent les territoires : aux origines des dispositifs dérogatoires de protection de l'enfance.}

\section{L'arrivée inégale des mineurs non accompagnés}

4 En France métropolitaine, l'arrivée des mineurs non accompagnés n'est pas un phénomène également réparti. Comme la majeure partie des flux migratoires, le voyage des jeunes se termine souvent dans la capitale, parfois dans des agglomérations situées près de frontières maritimes ou terrestres, et plus rarement dans des petites villes. En France, cette concentration des arrivées dans certains départements a conduit à un déséquilibre territorial. 
Illustration 1 - Nombre d'arrivées spontanées de jeunes évalués mineurs et isolés par département du 01/06/2013 au 31/05/2014

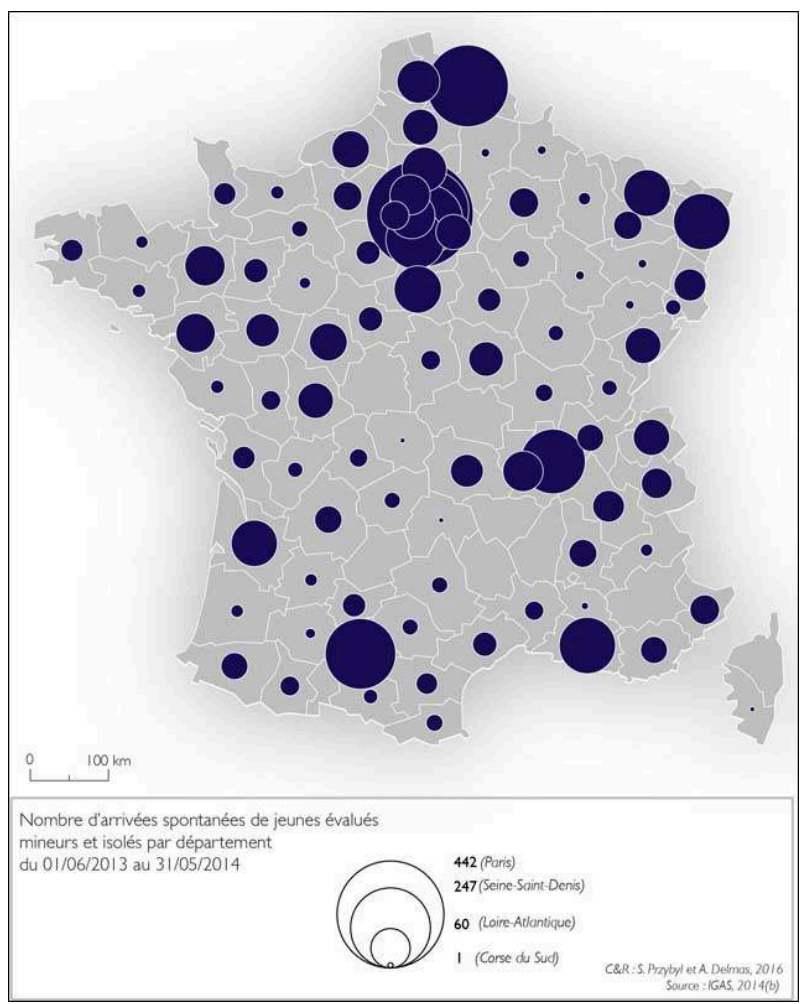

Source : IGAS, 2014b ; conception et réalisation : S. Przybyl et A. Delmas, 2016.

Devant l'obligation légale (article 375 du Code civil) de prise en charge des mineurs non accompagnés, certains territoires se sont retrouvés en difficulté au regard de l'absence de ressources financières, d'un manque d'infrastructures et d'une carence de moyens humains. Dans une conjoncture globale de baisse des financements accordés aux départements, la mission départementale de protection de l'enfance a été mise à mal. Dans ce contexte, la présence des mineurs non accompagnés en France a révélé autant qu'elle a confirmé toutes les limites de la position de «chef de file » endossée par les départements au gré des actes de la décentralisation en matière de protection de l'enfance. À ce sujet, le juriste Robert Lafore (2004, p. 21 et 27) qualifie les départements de "géants aux pieds d'argile " pour souligner toute l'ambivalence de ces territoires qui se sont vus octroyer d'importantes missions de protection et qui ont été dans le même temps fragilisés par ce transfert de compétences. En tant qu'enfant en danger, les mineurs non accompagnés répondent aux mêmes dispositions de droit commun prévus pour les nationaux. Toutefois, la situation inédite de saturation rencontrée par les services de protection de l'enfance a conduit à ce que de premiers dispositifs de prise en charge dérogatoires soient spécialement pensés pour ce public jugé atypique et prioritairement mis en place dans certains départements.

\section{Création et limites des premiers dispositifs dérogatoires à destination des mineurs non accompagnés}

6 Les premières initiatives destinées à accompagner les mineurs en dehors des circuits traditionnels de la protection de l'enfance sont nées dans le sud de la France. À 
Marseille, dès 1994, la création de l'association Jeunes Errants entend combler les lacunes de l'Aide sociale à l'enfance (ASE) et proposer un accompagnement à des jeunes en provenance des pays du Maghreb (Maroc et Algérie). À l'époque, les mineurs recueillis sont considérés comme étant de passage. Les moyens déployés apparaissent bien dérisoires comparés à ce que le droit commun prévoit traditionnellement pour des mineurs en danger. Cette association mise alors sur le rapatriement des mineurs en établissant un contact avec des familles restées au pays d'origine. Pour Jean-François Martini (2014, p.3), membre du Groupe d'information et de soutien des immigrés (GISTI), l'association Jeunes Errants ouvre la "première brèche dans le système de protection de l'enfance ». À Marseille, les jeunes accueillis n'entendent pourtant pas rentrer chez eux et s'imposent comme un nouveau défi pour les acteurs de la protection de l'enfance. Alors qu'à Marseille le dispositif s'ajuste face à cette nouvelle donne, la ville-département de Paris s'inspire du cas marseillais pour initier à son tour un dispositif dérogatoire quelques années plus tard.

$7 \mathrm{Au}$ début des années 2000 , les mineurs qui se présentent dans la ville de Paris augmentent. Selon le rapport établi par Angelina Etiemble (2002, p. 15), «ils seraient environ plus d'un millier en $2001 »$. Cette population interroge autant qu'elle pose problème à des institutions alors peu préparées et formées à l'accueil de ce public. À Paris comme à Marseille, ces mineurs perturbent le fonctionnement classique des services de l'ASE qui n'ont que peu de solutions à apporter à ces jeunes. Nombreux sont ceux qui se retrouvent forcés de survivre dans la rue, sans hébergement ni nourriture. Nombreux sont également ceux qui sont exposés aux violences de la rue et aux réseaux (traite, prostitution) (ibid., p. 81 et 89 ). Sur le terrain, des associations se mobilisent pour permettre un meilleur accès des mineurs au circuit de protection de droit commun. En 2001, l'association Soutien, solidarité et actions en faveur des émigrants (SSAÉ), Parada, la Cimade, France terre d'asile (FTDA) et d'autres proposent d'héberger et d'orienter ces jeunes vers les systèmes de protection. Cette mobilisation permet à un nombre croissant de mineurs d'être entendus par l'ASE. Néanmoins, manquant de moyens humains et financiers, les services de l'ASE croulent rapidement sous le nombre de demandes. Les jeunes quant à eux continuent d'arriver sur le territoire et se retrouvent sans solutions d'accueil. Les pouvoirs publics sont alertés par l'association SSAÉ et par des employés de l'ASE qui organisent une journée de grève symbolique pour réclamer plus de moyens et de meilleures conditions de travail.

8 À Paris, la situation apparaît de plus en plus inextricable et l'arrivée de mineurs non accompagnés continue d'exacerber les tensions. D'une part, les associations condamnent l'ASE et son absence de considération l'égard des mineurs; de l'autre, les services de l'ASE sont débordés et estiment que leur mission n'est pas de s'occuper des mineurs étrangers, mais des nationaux (Etiemble, 2002, p. 89). Les mineurs non accompagnés deviennent une "problématique sociale» (Burdillat, 2001, p. 15) qui alerte les pouvoirs publics. En septembre 2002, le premier dispositif dérogatoire à destination des mineurs non accompagnés est ainsi initié par l'État et mis en place à Paris. Appelé dispositif Versini, du nom de la secrétaire d'État chargée de la lutte contre la précarité et l'exclusion, il est entièrement financé par la Direction de l'action sociale et de l'enfance et de la santé (DASES) de la Mairie de Paris et l'État. Le dispositif entend proposer aux mineurs une mise à l'abri et un accueil dans des lieux spécialisés, ainsi qu'un accompagnement vers le droit commun (Bordin, 2008; Debré, 2010, p.14; Andreeva et al., 2013, p. 20). 
9 Les débuts du dispositif piloté par la DASES permettent d'améliorer la situation des mineurs présents dans la capitale, et de mieux répartir la mission de l'accueil entre les associations et l'ASE (Rongé, 2012, p. 22). Toutefois, compte tenu de l'arrivée toujours croissante de mineurs dans la capitale, le dispositif Versini se retrouve rapidement " sous-dimensionné » (Debré, 2011, p. 48). L'accueil physique des mineurs devant être de nouveau assuré par l'ASE, les jeunes se retrouvent encore une fois sans hébergement ni nourriture. Paris est plongé dans un « état de crise permanent » (Martini, 2012, p. 12) où la vie dans la rue est devenue une étape à part entière du parcours d'accueil et de protection.

\section{La réaffirmation d'un dispositif dérogatoire}

10 À mesure que les scandales éclatent et ternissent les engagements de la France en matière de protection de l'enfance, la situation parisienne force les différents acteurs à trouver une solution. Tout l'enjeu des autorités est de fluidifier ce nouveau circuit de prise en charge déjà grippé. Pour que l'ASE de Paris puisse accueillir les mineurs, le dispositif Versini est réaménagé, repensé et adapté. Si initialement la meilleure répartition des compétences de l'accueil avait permis de soulager l'ASE, la nouvelle version du dispositif transfère intégralement à trois associations la procédure d'accueil et de vérification de la minorité des mineurs qui prétendent au dispositif de protection de l'enfance. L'association Hors La Rue est chargée d'effectuer des maraudes pour repérer les mineurs en situation d'errance et propose des permanences dans ses locaux. La Croix-Rouge française assure la mise à l'abri, l'hébergement et le suivi éducatif des jeunes reconnus mineur et non accompagnés. L'association FTDA obtient quant à elle la plus grande part de ce marché et une responsabilité de taille en acceptant la mission centrale de l'évaluation de la minorité pour le compte de l'ASE (Martini, 2012 et 2014).

11 Dans le cadre de cette nouvelle redistribution de ces missions, la création de la Plateforme d'accueil et d'orientation des mineurs isolés étrangers (PAOMIE) gérée par FTDA est chargée de procéder à cette évaluation et devient alors l'unique voie d'accès à une protection. Les décisions rendues à la PAOMIE sont cruciales puisqu'elles ouvrent ou ferment les portes de l'ASE et donc, celles de la protection. Les méthodes employées pour l'évaluation de la minorité ne restent pas longtemps à la discrétion de l'association. Très rapidement, des témoignages viennent dénoncer les pratiques développées par FTDA.

«Dernière innovation en date: concéder à l'association France terre d'asile l'exclusivité du "premier accueil et l'orientation" des mineurs. FTDA a mis au point une procédure d'évaluation de l'âge qui fait la part belle à l'apparence physique des jeunes : taille, corpulence, voix, pilosité du visage, autant d'indices que l'association doit consigner sur une fiche d'évaluation, avant de saisir ou non les services de l'ASE. Celle-ci n'a plus besoin de procéder à un tri pénible, une association s'en charge maintenant à sa place ». Martini, 2012, p. 14.

12 À mesure que cette plateforme est devenue un maillon central de l'accueil des jeunes, elle ne s'est plus seulement contentée de se prononcer sur la minorité, mais a instauré ses propres critères de discrimination d'accès à la protection (âge minimum, apparence physique, etc.). D'un point de vue légal pourtant, tous les jeunes reconnus mineurs et en situation de danger devraient bénéficier d'une protection identique à celle octroyée aux enfants français (article $375 \mathrm{du}$ Code civil). Ces pratiques s'inscrivent en totale 
contradiction avec le principe de non-discrimination des mineurs de l'article 2 de la Convention internationale des droits de l'enfant (1989).

13 Le cas spécifique de la situation parisienne met au jour une logique de double décentralisation des compétences. La première s'est d'abord effectuée dans un mouvement allant de l'État vers les départements et s'inscrit dans le cadre global de délégation de la politique de protection de l'enfance. La seconde, spécifique aux mineurs non accompagnés, s'est instaurée entre les services départementaux de l'ASE et un ensemble d'associations. La délégation croissante et réticulaire des compétences à de nouveaux services a fait de la présence des mineurs non accompagnés plus qu'une problématique sociale reconnue, un véritable marché associatif concurrentiel.

\section{Les mineurs non accompagnés : d'une problématique locale à un enjeu national}

\section{La gronde des (autres) élus départementaux}

Longtemps, sur la question des mineurs non accompagnés, la ville-département de Paris a concentré toute l'attention politique et médiatique. Elle est devenue un laboratoire d'expérimentation de différents modèles d'accueil spécifiques s'éloignant des circuits traditionnels de prise en charge des enfants en danger. Rappelant le modèle de macrocéphalie que Jean-François Gravier détaillait dans son ouvrage Paris et le désert français en 1947, Paris est resté au fil des années l'espace le plus concerné par l'arrivée des mineurs mais aussi celui où de nombreux dispositifs inédits et ressources ont été mis en place (IGAS 2005, 2014(a) et (b) ; Debré, 2010).

Pourtant, dans le même temps, d'autres départements français se sont retrouvés également confrontés aux mêmes problématiques. L'attention suscitée par l'urgence parisienne a participé la mise sous silence de leur situation. Alors que la capitale voyait la mise en place de dispositions particulières, ces départements n'ont ni bénéficié d'une aide de l'État, ni de l'instauration de nouveaux modes d'accueil.

En septembre 2011, la décision d'un élu créée la polémique. Alors président du conseil départemental de la Seine-Saint-Denis, Claude Bartolone annonce dans un arrêté qu'il suspend l'accueil des mineurs non accompagnés se présentant dans son département. En raison de la présence de l'aéroport de Roissy, la Seine-Saint-Denis constitue une des portes d'entrée sur le territoire national et en fait un des espaces les plus concernés par l'arrivée de mineurs. À l'inverse de Paris, la Seine-Saint-Denis ne reçoit pourtant aucune aide spécifique de la part de l'État et manque cruellement de moyens. Sur son site officiel, l'élu explique toute sa détermination à obtenir une réponse du Gouvernement ${ }^{3}$.

«Pendant des années, j'ai alerté le Gouvernement sur les difficultés que rencontraient les services de l'aide à l'enfance de Seine-Saint-Denis dans l'accueil de mineurs isolés étrangers de plus en plus nombreux. [...] Il y a quatre semaines, j'ai mis l'État face à ses responsabilités en décidant de ne plus accueillir de nouveaux arrivants mineurs isolés étrangers au sein des services de l'aide sociale à l'enfance en Seine-Saint-Denis. [...] À ceux qui manient la désinformation, je le dis clairement: ce n'est pas maintenant que je faiblirai ou que je baisserai les bras [...] Les lignes ont enfin bougé, les citoyens ont pris la mesure d'un problème trop longtemps resté dans l'ombre médiatique, et les acteurs de terrain s'accordent sur la nécessité d'un changement ». Bartolone, 2011, www.infomie.net 
17 Sa prise de position trouve un écho d'autant plus important qu'il est soutenu par JeanPierre Rosenczveig, président du Tribunal de grande instance de Bobigny et personnage emblématique de la défense des droits des mineurs non accompagnés. Près d'un mois plus tard, au début du mois d'octobre 2011, Claude Bartolone obtient gain de cause. Un système de répartition est mis en place. Le département de la Seine-Saint-Denis n'accueille plus qu'un mineur sur les dix qui se présentent dans sa circonscription (Andreeva et al., 2013, p. 11). Les neuf autres sont envoyés dans d'autres départements français. Le choix de Claude Bartolone et le bras de fer qu'il entame avec l'État amorce de nombreux changements dans le paysage de la prise en charge des mineurs non accompagnés.

Quelques mois plus tard, l'accalmie négociée en région parisienne réveille la colère en province. Dans le sillage de Claude Bartolone, des présidents de conseils départementaux suspendent tour à tour l'accueil des mineurs. En effet, si Paris et la Seine-Saint-Denis sont concernés au premier chef, bien d'autres départements le sont eux aussi (illustration 1). À l'instar du Bas-Rhin et de la Mayenne, les voix des élus s'élèvent pour dénoncer le profond déséquilibre qui règne entre les départements français sur cette thématique. Réunis au sein de l'Association des départements de France (ADF), tous demandent une intervention urgente de l'État.

Les mineurs non accompagnés apparaissent alors comme un prétexte à la relance d'un dialogue avec le Gouvernement. Le regard porté au temps long de la décentralisation dans le domaine de la protection de l'enfance montre pourtant que les difficultés rencontrées par les collectivités sont davantage héritées qu'elles n'ont été créées par l'arrivée de ces jeunes (Przybyl, 2016). Outre la dénonciation des difficultés financières, certains départements exigent que l'État intervienne au titre de sa compétence dans la gestion des flux migratoires. Jusqu'alors cantonnée à la région parisienne, la situation de crise se diffuse à l'ensemble du territoire français. En tête, l'ADF est alors bien décidée à faire pression sur l'État et à obtenir une réponse comme Paris et la SeineSaint-Denis l'ont précédemment fait.

\section{La circulaire Taubira : une réponse d'abord politique?}

20 Le 31 mai 2013, les négociations entre l'ADF et l'État aboutissent à la publication de la " circulaire relative aux modalités de prise en charge des jeunes isolés étrangers: dispositif national de mise à l'abri, d'évaluation et d'orientation » par le ministère de la Justice. La circulaire entend répondre aux inquiétudes de certains départements et mettre fin à une situation de plus en plus délicate. Pour ce faire, le texte prévoit de limiter les disparités territoriales en matière d'arrivée des jeunes, de garantir les droits des mineurs et d'harmoniser les pratiques d'évaluation de la minorité à l'échelle nationale (Ministère de la Justice, 2013, p. 2).

21 Trois changements sont ainsi apportés au système d'accueil des mineurs non accompagnés sur le territoire français. Outre le financement des cinq premiers jours d'accueil et la diffusion d'une seule et unique trame d'évaluation de la minorité, le rééquilibrage géographique entend mettre fin aux déséquilibres territoriaux. Sur le modèle de répartition mis en place en Seine-Saint-Denis, l'État fait le choix de réorienter et redistribuer à l'échelle nationale les mineurs entre les différents départements à partir d'un indice statistique. C'est en fonction du nombre d'individus de moins de 19 ans par département que le rééquilibrage et l'orientation des mineurs 
sont décidés. Avec ce texte, l'État adresse un message de soutien attendu par les départements en posant des règles de fonctionnement de l'accueil communes à tous les acteurs. Sur le terrain, le texte est reçu par les professionnels comme un signal positif.

« Moi quand je l'ai lu j'étais content que l'État parle à nouveau de cette question-là et prenne ses responsabilités. Là-dessus, on ne peut pas le reprocher à Taubira. Voilà, l'État revient dans la danse. Alors après la manière dont il revient dans la danse, il s'avère que ça ne satisfait personne parce que là on est encore dans des paradoxes. Ces dispositifs-là sont jalonnés sans cesse par des paradoxes, parce qu'il y a des tensions entre compétences d'État et compétences de département ». Entretien T., éducateur spécialisé, 2014.

Pour la sociologue Martine Burdillat (2001), la prise de position d'un État par l'intermédiaire d'une loi ou d'une circulaire, c'est-à-dire une norme de rang légal ou réglementaire, marque l'étape ultime de la reconnaissance d'un phénomène en problématique et donne lieu à l'instauration d'un traitement spécifique. Ainsi, après la création des premiers dispositifs dérogatoires en région parisienne, la mise en application de la circulaire de mai 2013 marque l'aboutissement de l'instauration d'un traitement particulier à l'égard de cette population à l'échelle nationale. Les nombreux débats et négociations érigent les mineurs non accompagnés en une problématique nationale.

\section{Les limites de la redistribution des mineurs non accompagnés}

Originellement, mettre fin aux disparités territoriales de l'accueil des mineurs constitue une des demandes les plus pressantes des présidents de conseil départemental. En voulant mettre fin à ce déséquilibre par la circulaire, l'État endosse une de ses plus anciennes compétences de régulateur et de garant du bon équilibre territorial. Les premières difficultés et limites du modèle de répartition apparaissent au moment de la mise en pratique de l'indice statistique. Si la circulaire semblait avoir cerné les enjeux des départements les plus concernés, le texte n'a pas tenu compte des capacités d'accueil des nouveaux départements d'arrivée des mineurs non accompagnés. Lors de la mise en application du texte, certaines localités ont dû recevoir des mineurs quand bien même elles ne disposaient ni des structures disponibles ni des moyens humains pour les accompagner. Suite à l'arrivée de seulement quelques jeunes, la situation de saturation d'espaces d'accueil a été de nouveau atteinte.

"Les départements commencent aussi à se poser des questions sur ce qu'on leur demande de faire, ou de financer. [...] Et puis petit à petit, tous les départements vont atteindre un seuil où, quotas ou pas quotas, ils vont dire "stop c'est bon on n'en peut plus " ». Entretien D., directeur association, 2014.

En difficulté, ces territoires se sont retrouvés dans la même situation que ceux qui tiraient la sonnette d'alarme quelques mois plus tôt. En voulant rééquilibrer les disparités territoriales, la circulaire a réamorcé avec elle la spirale de l'urgence et de la négociation. Ces nouvelles situations de saturation des dispositifs de protection ont provoqué de nouvelles suspensions de l'accueil des mineurs. Les Alpes-Maritimes, l'Aveyron, la Corse-du-Sud, la Côte-d'Or, l'Eure-et-Loir, le Loir-et-Cher, le Loiret, la Sarthe, la Vendée et les Hauts-de-Seine ont alors saisi le Conseil d'État pour exiger une révision de la clé de répartition des mineurs inscrite de la circulaire. Le 30 janvier 2015, le Conseil d'État rend son avis et impose une annulation partielle du texte. Sans remettre en cause le principe de répartition entre les départements, l'autorité invalide 
le recours à l'indice statistique en soulignant notamment que ce type de mesure devrait être inscrit dans une loi et non régie par une simple circulaire. Un document complémentaire juridique explique que le placement des mineurs non accompagnés doit désormais tenir compte du nombre de places disponibles dans les départements et réaffirme également le principe de l'intérêt supérieur de l'enfant dans cette procédure (Ministère de la Justice, 2015).

À l'échelle de plusieurs villes, de quelques départements et désormais à l'échelle de tous les départements, la régulation de la présence des mineurs a fait des mineurs non accompagnés une problématique résolument nationale. Comme un témoignage de cette diffusion du phénomène à l'ensemble du territoire, les derniers chiffres fournis par sur le site du ministère de la Justice en 2016 (www.justice-gouv.fr) révèlent le nombre de mineurs non accompagnés confiés par décision de justice dans chaque département après l'application de la nouvelle clé de répartition. À partir des données fournies par le ministère, la carte de l'illustration 2 montre qu'en dépit d'une présence toujours prononcée dans certains espaces, la présence des mineurs accompagnés s'étend désormais à l'ensemble des localités françaises et concerne tous les départements.

Illustration 2 - Nombre de mineurs non accompagnés par département confiés par décisions judiciaires au 23 septembre 2016

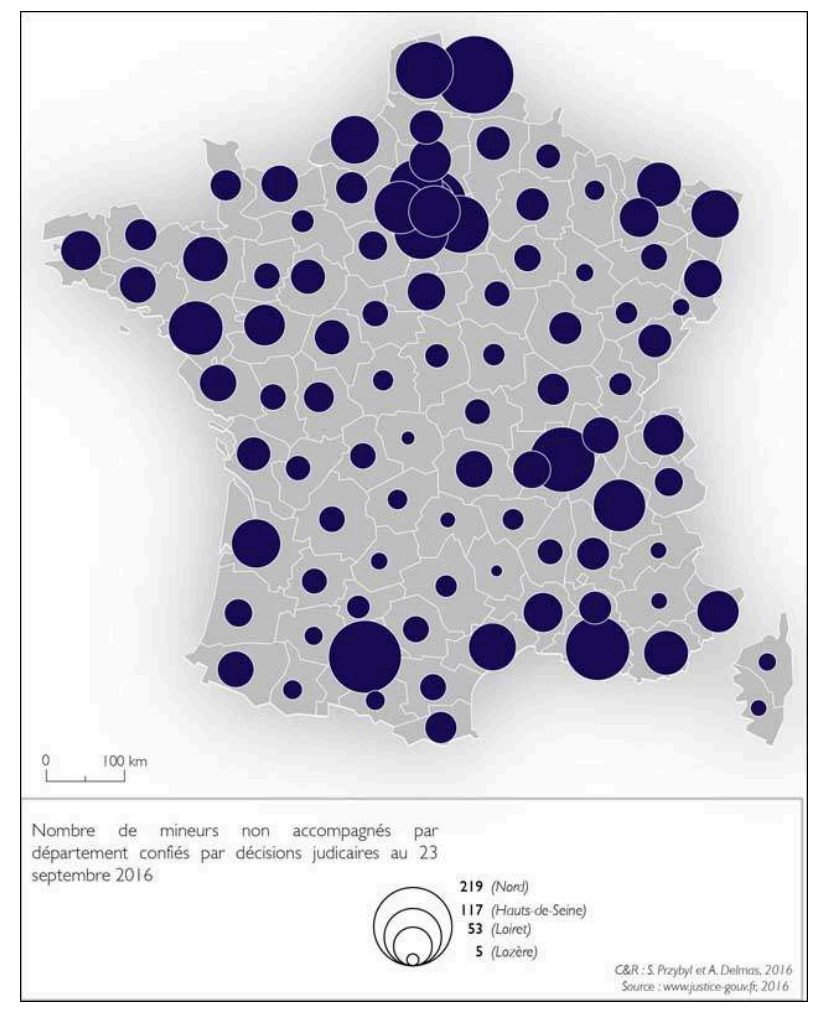

Source : www.justice-gouv.fr, 2016 ; conception et réalisation : S. Przybyl et A. Delmas, 2016.

\section{Quelle place pour les droits fondamentaux des mineurs non accompagnés?}

Dans ce climat de tension permanente, les préoccupations institutionnelles des départements ont réglementé le parcours des mineurs non accompagnés. L'intensité 
croissante des négociations entre les associations, les départements et l'État a progressivement mis dans l'ombre les mineurs, pourtant premiers concernés par ces mutations. En effet, au fil du texte de la circulaire de mai 2013, l'accent est davantage porté aux acteurs qui doivent se charger des jeunes plus que sur les mineurs euxmêmes. Ce premier constat relatif à la circulaire va à l'encontre du principe de l'intérêt supérieur de l'enfant évoqué comme une « considération primordiale » dans l'article 3 de la Convention internationale des droits de l'enfant (1989).

«Peut-être que c'est le premier déclencheur de quelque chose qui va être plus intéressant pour les mineurs parce que là, cette procédure elle est intéressante que pour les départements. Il n'y a aucun intérêt pour les mineurs à ce qu'ils subissent cette procédure. C'est une histoire de " dispatchage " des jeunes créée pour faire plaisir à certains départements saturés. Le jeune, et l'intérêt supérieur de l'enfant il n'a pas été abordé un quart de seconde dans cette circulaire. Il a peut-être été réfléchi, mais concrètement, je ne vois pas où est l'intérêt supérieur de l'enfant au moment de lui faire subir ce type de procédure». Entretien D., directeur association, 2014.

L'absence de considération pour les mineurs est plus particulièrement prégnante dans la partie dédiée à leur réorientation vers de nouveaux départements. La description de la redistribution est d'abord évoquée dans sa dimension financière (Ministère de la Justice, 2013, p. 4) avant que l'importance de la rapidité de traitement des dossiers des mineurs soit abordée comme un principe clé de cette procédure (ibid, p. 5). Enfin, en concluant « qu'il n'y a pas de critère législatif qui guide l'orientation du jeune si ce n'est celui de la clé d'orientation » (ibid., p. 6), la circulaire confirme que l'intérêt supérieur de l'enfant est loin d'être une considération primordiale de ce nouveau modèle.

Tel qu'il a été pensé, le dispositif de répartition prône davantage le respect de la souveraineté des départements que celui de l'intérêt supérieur de l'enfant. La logique d'orientation des mineurs tient plutôt compte des intérêts des départements à devoir accueillir des jeunes plus qu'il ne considère celui des mineurs à se trouver dans tel ou tel département. Ce dispositif d'accueil des mineurs non accompagnés est une preuve de l'introduction d'une logique gestionnaire dans les principes de la protection de l'enfance. Comme une confirmation de cette limite, la décision rendue par le Conseil d'État en 2015 rappelle la nécessité d'intégrer avec plus de force le principe de l'intérêt supérieur de l'enfant dans les décisions de réorientation des mineurs non accompagnés sur le territoire. Le Conseil d'état invite dès lors les Parquets à s'assurer de l'existence de conditions satisfaisantes pour apprécier la capacité des départements à accueillir les mineurs et dépasser ainsi une logique gestionnaire résumée à la seule variable des places disponibles. Pour Jean-Pierre Rosenczveig qui soutenait l'initiative de Claude Bartolone en 2011, l'amplification de la répartition a fait de ces mineurs des " paquets » que l'État déplace à sa guise (www.jprosen.blog.lemonde.fr). Les mineurs non accompagnés sont devenus une sorte de variable d'ajustement dans les négociations entre les collectivités.

En ouvrant la voie au déplacement des mineurs sur le territoire, la circulaire confirme la condition d'étranger des mineurs telle que décrite par Georg Simmel en 1908 dans son texte Digressions sur l'étranger (Simmel, 1908). Le sociologue indiquait que le propre de l'étranger est d'être sans attaches, sans aucune propriété ; dans cette perspective, il peut alors se déplacer et être déplacé sans limites. Avec ce principe nouveau d'orientation, les mineurs non accompagnés apparaissent comme des individus hors des lieux, complètement dépourvus de liens ou de contacts. S'il est vrai que certains 
mineurs arrivent en France sans avoir réellement une idée du lieu où ils sont, d'autres, à l'inverse, ne sont pas là par hasard.

«- Quand tu es revenu en France tu as dormi à la gare de l'Est aussi ? On t'a aidé ?

- Je suis resté encore un mois là-bas oui.

- Tout seul sans rien?

- Non pas tout seul, j'étais avec des amis [...]». Entretien A., 16 ans, Afghanistan.

Paris, 2012.

Comme le témoignage de A. le souligne, des jeunes arrivent dans certaines villes en raison de la présence de membres de leur communauté d'origine, de leur famille, d'amis ou de proches déjà installés. Ces individus constituent un réseau de solidarité potentiel ou bien réel sur lequel les jeunes vont pouvoir s'appuyer lors de leur parcours en France. En quête d'ancrage, de sécurité et de stabilité, le départ vers un département inconnu peut être perçu comme un véritable déchirement.

«Moi ils m'ont mis là-bas, mais je n'aime pas les montagnes. En plus il fait trop froid. Il n'y a rien, même pas de magasins ! C'est trop vide ! À Paris, c'est mieux, il y a du bruit, des filles, des voitures et moi je préfère ça. Je ne veux pas repartir là-bas c'est vraiment pas bien». Extrait carnet de terrain, discussion avec A., 17 ans, Pakistan. Paris, 2012.

«À Albi j'étais comme chez moi. Je me sentais à l'aise là-bas. Là-bas aucun éducateur n'est venu me dire que je n'étais pas chez moi. Là-bas, je ne sortais même pas du foyer. Je ne voulais pas sortir, car je n'en voyais pas l'importance. Tout autour de moi il y avait toujours des gens qui me montrent de l'amour, tu vois ce que je veux dire? ». Entretien J., 15 ans, Angola. Saverdun, 2014.

Pour mettre fin à une inégalité territoriale et garantir la souveraineté des collectivités, la circulaire consent ainsi à taire l'intérêt supérieur de l'enfant, un élément pourtant crucial de toute décision qui concerne les jeunes. Dans ce contexte de mise à mal des droits fondamentaux, l'avis rendu par la Commission nationale consultative des droits de l'Homme (CNCDH) en 2014 sur la situation des mineurs non accompagnés présents en France rappelle les autorités à leurs responsabilités. Son constat est sans appel. Les dispositions prises en France ne respectent pas les droits des mineurs, pire encore, elles entravent leur protection. L'avis de la CNCDH condamne fermement les évolutions réglementaires et invite les autorités à reprendre en main le dossier des mineurs non accompagnés en veillant au respect de leurs droits.

\section{Conclusion : mineurs en danger, mineurs à contrôler...}

Le jeu de négociations entre État et département dans les évolutions de l'accueil des mineurs non accompagnés a rendu les frontières poreuses entre la protection de l'enfance (compétence départementale) et le contrôle des flux migratoires (compétence étatique). Les considérations pour la régulation de cette population ont progressivement pris le pas sur l'effectivité de sa protection et s'invitent désormais comme une nouvelle variable dans le contexte de leur prise en charge. Les débats accrus autour du choix de l'acteur compétent n'ont d'ailleurs cessé de puiser leur légitimité dans la situation de ces mineurs non accompagnés jugés à mi-chemin entre deux champs de compétences. L'intervention de l'État a entériné l'appartenance des mineurs au champ de la gestion des migrations internationales. À cet égard, la CNCDH soulignait en 2014 le risque représenté par cette intervention et rappelait que les mineurs sont d'abord une compétence des départements. 
«Le signal envoyé par la mise en place de ce nouveau protocole est fort: l'État reconnait sa responsabilité à l'égard d'une question qui relève normalement de la compétence des départements. À cet égard, la CNCDH entend néanmoins préciser que cette intervention de l'État ne doit en aucun cas aboutir à aborder la problématique [...] sous le seul angle de la gestion des flux migratoires [...]». CNCDH, 2014.

Près de trois ans plus tard, le 20 octobre 2017, le premier ministre Édouard Philippe annonçait lors d'un congrès national réunissant les présidents des conseils départementaux une nouvelle intervention de l'État sur la question des mineurs non accompagnés. À Marseille, évoquant un phénomène "plus massif et diffus » et une situation intenable, Édouard Philippe a assuré aux élus que l'évaluation de la minorité des jeunes serait désormais entièrement assumée par les services de l'État et ne serait plus à la charge des structures départementales. En dépit des avertissements posés par la CNCDH et des nombreuses alertes du Défenseur des droits sur l'appartenance des mineurs non accompagnés au champ de la protection de l'enfance en danger, cette nouvelle mesure réaffirme l'orientation des mineurs vers un circuit d'accueil relevant de la gestion des flux migratoires par l'État. Dans cette dynamique, l'État revêt de nouveau son rôle de garant du bon équilibre des territoires. Face à cette annonce qui semble satisfaire les élus départementaux, de nouvelles voix s'élèvent. Associations et défenseurs des droits des enfants publient des communiqués pour témoigner de leur inquiétude quant au respect de l'intérêt supérieur de ces enfants avant toute chose en situation de danger. Près de quatre ans après la mise en place de la circulaire Taubira cette annonce interroge : quel sera le nouveau modèle territorial dérogatoire de gestion des mineurs non accompagnés?

\section{BIBLIOGRAPHIE}

Andreeva M., Légaut J-P., Tawfik L., Lardanchet G. et Senovilla Hernández D., 2013. Mineurs isolés étrangers et sans protection en Europe. Recherche conduite en France dans le cadre du projet PUCAFREU. PUCAFREU - Promoting unaccompanied children's access to fundamental rights in the European Union, Poitiers, 96 p.

Bordin D., 2008. France Terre d'Asile et le dispositif parisien face aux mineurs isolés en transit. Journal du droit des jeunes, vol. 7, n² 277, p. 28-32.

Burdillat M., 2001. L'administration sanitaire et sociale dans le champ du débat politique et social. Revue française des affaires sociales, vol. 4, $\mathrm{n}^{\circ}$ 4, p. 13-31.

Debré I., 2010. Les mineurs isolés étrangers en France. Paris, Sénat, 160 p.

Etiemble A., 2002. Les mineurs isolés étrangers en France. Évolution quantitative de la population accueillie à l'Aide Sociale à l'Enfance. Les termes de l'accueil et de la prise en charge. Étude réalisée pour la Direction de la Population et des Migrations. Rennes, QUEST'US, $272 \mathrm{p}$.

Gravier J-F., 1947. Paris et le désert français. Paris, Flammarion, 284 p. 
Inspection générale des affaires sociales (IGAS), 2005. Mission d'analyse et de proposition sur les conditions d'accueil des mineurs étrangers isolés en France. Paris, $114 \mathrm{p}$.

Inspection générale des affaires sociales (IGAS), 2014(a). L'évaluation du dispositif relatif aux mineurs isolés étrangers mis en place par le protocole et la circulaire du 31 mai 2013. Paris, 176 p.

Inspection générale des affaires sociales (IGAS), 2014(b). L'évaluation du dispositif relatif aux mineurs isolés étrangers mis en place par le protocole et la circulaire du 31 mai 2013. Annexes, Paris, 86 p.

Lafore R., 2004. La décentralisation de l'action sociale. L'irrésistible ascension du « département providence ». Revue française des affaires sociales, vol. 4, numéro 4, p. 17-34.

Martini J.-F., 2012. Mineurs étrangers : le tri qui tue. Plein droit, vol. 1, nº 92, p. 11-15.

Martini J.-F., 2014. Allez vous faire protéger ailleurs! Plein droit, vol. 3, nº102, p. 3-8.

Ministère de la justice, 2013. Circulaire relative aux modalités de prise en charge des jeunes isolés étrangers : dispositif national de mise à l'abri, d'évaluation et d'orientation. Paris, Ministère de la Justice, $6 \mathrm{p}$.

Ministère de la justice, 2015. Modalités de prise en charge des mineurs isolés étrangers suite à la décision du Conseil d'État du 30 janvier 2015 portant sur la légalité de la circulaire du 31 mai 2013 relative aux modalités de prise en charge des jeunes isolés étrangers : dispositif nation de mise à l'abri, d'évaluation et d'orientation. Paris, Ministère de la Justice, 3 p.

Ministère de la justice, 2017. Rapport annuel d'activité 2016. Mission mineurs non accompagnés. Paris, Ministère de la Justice, $27 \mathrm{p}$.

Przybyl S., 2016. Territoires de la migration, territoires de la protection. Parcours et expériences des mineurs isolés étrangers accueillis en France. Thèse de géographie, sous la direction de William Berthomière et Daniel Senovilla Hernández, Université de Poitiers, 515 p.

Rongé J.-L., 2012. Une absence volontaire de protection : les mineurs isolés étrangers victimes de maltraitance institutionnelle. Journal du droit des jeunes, $\mathrm{n}^{\circ}$ 311, p. 19-24.

Simmel G., 1908. Digressions sur l'étranger. In Grafmeyer Y., Joseph I., 2004. L'École de Chicago : naissance de l'écologie urbaine, Paris, Flammarion, p. 53- 59

Terrier E., Halifax J., 2017, Approche territoriale de la protection de l'enfance. Quelles spécificités des espaces urbains, ruraux et périurbains? Le sociographe, vol. 5, n 10, p. 61-82

\section{NOTES}

1. Depuis 2016 et l'intervention du Garde des sceaux, l'appellation de mineur isolé étranger a laissé place à celle de mineur non accompagné. Les différentes sources bibliographiques mobilisent la terminologie de mineur isolé étranger.

2. Dans leur grande majorité, les entretiens semi-directifs ont été réalisés en français. Certaines précisions ou parties ont pu être conduites en anglais en cas de besoin ou d'incompréhension.

3. Supprimé du site officiel de Claude Bartolone depuis, le contenu du billet reste disponible sur le site InfoMie. 


\section{RÉSUMÉS}

Depuis trente ans, la France est confrontée à l'arrivée croissante des mineurs non accompagnés. Âgés de moins de 18 ans et se trouvant sur le territoire sans leur représentant légal, ces jeunes se trouvent à mi-chemin entre les considérations pour la protection de l'enfance et celles de la gestion de l'immigration clandestine. Au fil du temps, plus qu'un problème politique, leur répartition très hétérogène au sein des départements français est devenue un réel enjeu géographique. Sur fonds de négociations politiques, cet article revient sur les différentes décisions prises par les autorités pour rétablir l'équilibre territorial et interroge la place qui a été réservée à l'intérêt supérieur de l'enfant.

For thirty years, France has been confronted with the increasing arrival of unaccompanied minors. Under 18 years of age, they are in the territory without their legal representative. These young people are halfway between considerations for child protection and those of managing illegal immigration. Over time, more than a political problem, their very heterogeneous distribution within the French departments has become a real geographical issue. On the basis of political negotiations, this article reviews the decisions taken by the authorities to restore territorial balance and questions the place reserved for the best interests of the child.

\section{INDEX}

Mots-clés : mineur non accompagné, territoire, protection de l'enfance, intérêt supérieur de l'enfant, immigration

Keywords : unaccompanied minor, territory, child protection, best interests of the child, immigration

\section{AUTEUR}

\section{SARAH PRZYBYL}

Sarah Przybyl, sarah.przybyl@univ-poitiers.fr, est Géographe, Post-doctorante programme CAMIGRI, UMR MIGRINTER - Université de Poitiers. Elle a publié récemment :

- Przybyl S., 2016. Territoires de la migration, territoires de la protection. Parcours et expériences des mineurs isolés étrangers accueillis en France. Thèse de géographie, sous la direction de William Berthomière et Daniel Senovilla Hernández, Université de Poitiers, 515 p.

- Ben Tayeb Y. et Przybyl S., 2013. Tanger et les harragas : les mutations d'un espace frontalier. Hommes et migrations, $\mathrm{n}^{\circ} 1304$, p. 41-48. 